\title{
INFINITE FAMILIES OF RECURSIVE FORMULAS GENERATING POWER MOMENTS OF TERNARY KLOOSTERMAN SUMS WITH SQUARE ARGUMENTS ASSOCIATED WITH $O^{-}(2 n, q)$
}

\author{
DAE SAN Kim
}

\begin{abstract}
In this paper, we construct eight infinite families of ternary linear codes associated with double cosets with respect to certain maximal parabolic subgroup of the special orthogonal group $S O^{-}(2 n, q)$. Here $q$ is a power of three. Then we obtain four infinite families of recursive formulas for power moments of Kloosterman sums with square arguments and four infinite families of recursive formulas for even power moments of those in terms of the frequencies of weights in the codes. This is done via Pless power moment identity and by utilizing the explicit expressions of exponential sums over those double cosets related to the evaluations of "Gauss sums" for the orthogonal groups $O^{-}(2 n, q)$.
\end{abstract}

\section{Introduction}

Let $\psi$ be a nontrivial additive character of the finite field $\mathbb{F}_{q}$ with $q=p^{r}$ elements ( $p$ a prime). Then the Kloosterman sum $K(\psi ; a)([13])$ is defined by

$$
K(\psi ; a)=\sum_{\alpha \in \mathbb{F}_{q}^{*}} \psi\left(\alpha+a \alpha^{-1}\right)\left(a \in \mathbb{F}_{q}^{*}\right) .
$$

For this, we have the Weil bound

$$
|K(\psi ; a)| \leq 2 \sqrt{q} .
$$

The Kloosterman sum was introduced in 1926 ([12]) to give an estimate for the Fourier coefficients of modular forms.

Received September 11, 2009.

2010 Mathematics Subject Classification. 11T23, 20G40, 94B05.

Key words and phrases. index terms-Kloosterman sum, orthogonal group, special orthogonal group, double cosets, maximal parabolic subgroup, Pless power moment identity, weight distribution.

This work was supported by National Foundation of Korea Grant funded by the Korean Government(2009-0072514). 
For each nonnegative integer $h$, by $M K(\psi)^{h}$ we will denote the $h$-th moment of the Kloosterman sum $K(\psi ; a)$. Namely, it is given by

$$
M K(\psi)^{h}=\sum_{a \in \mathbb{F}_{q}^{*}} K(\psi ; a)^{h} .
$$

If $\psi=\lambda$ is the canonical additive character of $\mathbb{F}_{q}$, then $M K(\lambda)^{h}$ will be simply denoted by $M K^{h}$.

Also, we introduce an incomplete power moments of Kloosterman sums. Namely, for every nonnegative integer $h$, and $\psi$ as before, we define

$$
S K(\psi)^{h}=\sum_{a \in \mathbb{F}_{q}^{*}, \text { a square }} K(\psi ; a)^{h},
$$

which is called the $h$-th moment of Kloosterman sums with "square arguments". If $\psi=\lambda$ is the canonical additive character of $\mathbb{F}_{q}$, then $S K(\lambda)^{h}$ will be denoted by $S K^{h}$, for brevity.

Explicit computations on power moments of Kloosterman sums were begun with the paper [18] of Salié in 1931, where he showed, for any odd prime $q$,

$$
M K^{h}=q^{2} M_{h-1}-(q-1)^{h-1}+2(-1)^{h-1}(h \geq 1) .
$$

Here $M_{0}=0$, and, for $h \in \mathbb{Z}_{>0}$,

$$
M_{h}=\left|\left\{\left(\alpha_{1}, \ldots, \alpha_{h}\right) \in\left(\mathbb{F}_{q}^{*}\right)^{h} \mid \sum_{j=1}^{h} \alpha_{j}=1=\sum_{j=1}^{h} \alpha_{j}^{-1}\right\}\right| .
$$

For $q=p$ odd prime, Salié obtained $M K^{1}, M K^{2}, M K^{3}, M K^{4}$ in [18] by determining $M_{1}, M_{2}, M_{3}$. On the other hand, $M K^{5}$ can be expressed in terms of the $p$-th eigenvalue for a weight 3 newform on $\Gamma_{0}(15)$ (cf. [14], [17]). $M K^{6}$ can be expressed in terms of the $p$-th eigenvalue for a weight 4 newform on $\Gamma_{0}(6)$ (cf. [3]). Also, based on numerical evidence, in [1] Evans was led to propose a conjecture which expresses $M K^{7}$ in terms of Hecke eigenvalues for a weight 3 newform on $\Gamma_{0}(525)$ with quartic nebentypus of conductor 105 .

Assume from now on that $q=3^{r}$. Recently, Moisio was able to find explicit expressions of $M K^{h}$ for $h \leq 10$ (cf. [16]). This was done, via Pless power moment identity, by connecting moments of Kloosterman sums and the frequencies of weights in the ternary Melas code of length $q-1$, which were known by the work of Geer, Schoof, and Vlugt in [2]. In [9], we were able to produce two recursive formulas generating power moments of Kloosterman sums with square arguments and one recursive formula generating even power moments of those. To do that, we constructed three ternary linear codes $C\left(S O^{-}(2, q)\right)$, $C\left(O^{-}(2, q)\right), C\left(S O^{-}(4, q)\right)$, respectively associated with the orthogonal groups $S O^{-}(2, q), O^{-}(2, q), S O^{-}(4, q)$, and express those power moments in terms of the frequencies of weights in each code. In [11], the symplectic groups $S p(2, q)$ and $S p(4, q)$ were used instead in order to produce recursive formulas generating power moments and even power moments of Kloosterman sums with square arguments. 
In this paper, we will be able to produce four infinite families of recursive formulas generating power moments of Kloosterman sums with square arguments and four infinite families of recursive formulas generating even power moments of those. To do that, we construct eight infinite families of ternary linear codes $C\left(D C_{1}^{+}(n, q)\right)(n=2,4, \ldots), C\left(D C_{1}^{-}(n, q)\right)(n=1,3, \ldots)$, both associated with $Q \sigma_{n-1} Q ; C\left(D C_{2}^{+}(n, q)\right)(n=2,4, \cdots), C\left(D C_{2}^{-}(n, q)\right)(n=3,5, \ldots)$ both associated with $Q \sigma_{n-2} Q ; C\left(D C_{3}^{+}(n, q)\right)(n=2,4, \ldots), C\left(D C_{3}^{-}(n, q)\right)$ $(n=3,5, \ldots)$ both associated with $\rho Q \sigma_{n-2} Q ; C\left(D C_{4}^{+}(n, q)\right)(n=4,6, \ldots)$, $C\left(D C_{4}^{-}(n, q)\right)(n=3,5, \ldots)$ both associated with $\rho Q \sigma_{n-3} Q$, with respect to the maximal parabolic subgroup $Q=Q(2 n, q)$ of the special orthogonal group $S O^{-}(2 n, q)$, and express those power moments in terms of the frequencies of weights in each code. Then, thanks to our previous results on the explicit expressions of exponential sums over those double cosets related to the evaluations of "Gauss sums" for the orthogonal groups $O^{-}(2 n, q)[4,5]$, we can express the weight of each codeword in the duals of the codes in terms of Kloosterman sums or squares of Kloosterman sums. Then our formulas will follow immediately from the Pless power moment identity. Analogously to these, in [8], we obtained infinite families of recursive formulas for power moments of Kloosterman sums with square arguments and for even power moments of those by constructing ternary linear codes associated with double cosets with respect to certain maximal parabolic subgroup of the symplectic group $S p(2 n, q)$.

Theorem 1.1 in the following (cf. (1.19), (1.20), (1.22)-(1.25)) is the main result of this paper. Henceforth, we agree that, for nonnegative integers $a, b$, $c$,

$$
\left(\begin{array}{c}
c \\
a, b
\end{array}\right)=\frac{c !}{a ! b !(c-a-b) !}, \text { if } a+b \leq c
$$

and

$$
\left(\begin{array}{c}
c \\
a, b
\end{array}\right)=0, \text { if } a+b>c .
$$

To simplify notations, we introduce the following ones which will be used throughout this paper at various places.

$$
\begin{gathered}
A_{1}^{+}(n, q)=q^{\frac{1}{4}\left(5 n^{2}-2 n-4\right)}\left(q^{n-1}-1\right) \prod_{j=1}^{(n-2) / 2}\left(q^{2 j-1}-1\right), \\
B_{1}^{+}(n, q)=(q+1) q^{\frac{1}{4} n^{2}} \prod_{j=1}^{(n-2) / 2}\left(q^{2 j}-1\right), \\
A_{2}^{+}(n, q)=q^{\frac{1}{4}\left(5 n^{2}-2 n-8\right)}\left[\begin{array}{c}
n-1 \\
1
\end{array}\right]_{q} \prod_{j=1}^{(n-2) / 2}\left(q^{2 j-1}-1\right),
\end{gathered}
$$




$$
\begin{gathered}
B_{2}^{+}(n, q)=(q+1) q^{\frac{1}{4}(n-2)^{2}}\left(q^{n-1}-1\right) \prod_{j=1}^{(n-2) / 2}\left(q^{2 j}-1\right), \\
A_{3}^{+}(n, q)=(q+1) q^{\frac{1}{4}\left(5 n^{2}-2 n-8\right)}\left[\begin{array}{c}
n-1 \\
1
\end{array}\right]_{q} \prod_{j=1}^{(n-2) / 2}\left(q^{2 j-1}-1\right), \\
B_{3}^{+}(n, q)=q^{\frac{1}{4}(n-2)^{2}}\left(q^{n-1}-1\right) \prod_{j=1}^{(n-2) / 2}\left(q^{2 j}-1\right), \\
A_{4}^{+}(n, q)=(q+1) q^{\frac{1}{4}\left(5 n^{2}-6 n-4\right)}\left[\begin{array}{c}
n-1 \\
2
\end{array}\right]_{q} \prod_{j=1}^{(n-2) / 2}\left(q^{2 j-1}-1\right),
\end{gathered}
$$

$$
\begin{aligned}
& B_{4}^{+}(n, q)=q^{\frac{1}{4}(n-2)^{2}}\left(q^{n-1}-1\right) \prod_{j=1}^{(n-2) / 2}\left(q^{2 j}-1\right) \\
& A_{1}^{-}(n, q)=q^{\frac{5}{4}\left(n^{2}-1\right)} \prod_{j=1}^{(n-1) / 2}\left(q^{2 j-1}-1\right) \\
& B_{1}^{-}(n, q)=(q+1) q^{\frac{1}{4}(n-1)^{2}} \prod_{j=1}^{(n-1) / 2}\left(q^{2 j}-1\right), \\
& A_{2}^{-}(n, q)=q^{\frac{1}{4}\left(5 n^{2}-4 n-5\right)}\left[\begin{array}{c}
n-1 \\
1
\end{array}\right]_{q} \prod_{j=1}^{(n-1) / 2}\left(q^{2 j-1}-1\right), \\
& B_{2}^{-}(n, q)=(q+1) q^{\frac{1}{4}(n-1)^{2}} \prod_{j=1}^{(n-1) / 2}\left(q^{2 j}-1\right), \\
& A_{3}^{-}(n, q)=(q+1) q^{\frac{1}{4}\left(5 n^{2}-4 n-5\right)}\left[\begin{array}{c}
n-1 \\
1
\end{array}\right]_{q} \prod_{j=1}^{(n-1) / 2}\left(q^{2 j-1}-1\right) \text {, } \\
& B_{3}^{-}(n, q)=q^{\frac{1}{4}(n-1)^{2}} \prod_{j=1}^{(n-1) / 2}\left(q^{2 j}-1\right) \\
& A_{4}^{-}(n, q)=(q+1) q^{\frac{1}{4}\left(5 n^{2}-4 n-9\right)}\left[\begin{array}{c}
n-1 \\
2
\end{array}\right]_{q} \prod_{j=1}^{(n-3) / 2}\left(q^{2 j-1}-1\right)
\end{aligned}
$$




$$
B_{4}^{-}(n, q)=q^{\frac{1}{4}(n-3)^{2}}\left(q^{n-2}-1\right)\left(q^{n-1}-1\right) \prod_{j=1}^{(n-3) / 2}\left(q^{2 j}-1\right) .
$$

From now on, it is assumed that either +signs or - signs are chosen everywhere, whenever \pm signs appear.

Theorem 1.1. Let $q=3^{r}$. Then with the notations in (1.3)-(1.18), we have the following.

(1) With $i=1,3$, and + signs everywhere for \pm signs, we have a recursive formula generating power moments of Kloosterman sums with square arguments over $\mathbb{F}_{q}$ (cf. (1.2)) for each $n \geq 2$ even and all $q$; with $i=1$ and - signs everywhere for \pm signs, we have such a formula for each $n \geq 1$ odd and all $q$; with $i=3$ and - signs everywhere for \pm signs, we have such a formula for each $n \geq 3$ odd and all $q$.

$$
\begin{aligned}
& ( \pm(-1))^{h} S K^{h} \\
= & -\sum_{l=0}^{h-1}( \pm(-1))^{l}\left(\begin{array}{c}
h \\
l
\end{array}\right) B_{i}^{ \pm}(n, q)^{h-l} S K^{l} \\
& +q A_{i}^{ \pm}(n, q)^{-h} \sum_{j=0}^{\min \left\{N_{i}^{ \pm}(n, q), h\right\}}(-1)^{j} C_{i, j}^{ \pm}(n, q) \\
& \times \sum_{t=j}^{h} t ! S(h, t) 3^{h-t} 2^{t-h-j-1}\left(\begin{array}{c}
N_{i}^{ \pm}(n, q)-j \\
N_{i}^{ \pm}(n, q)-t
\end{array}\right)(h=1,2, \ldots),
\end{aligned}
$$

where $N_{i}^{ \pm}(n, q)=\left|D C_{i}^{ \pm}(n, q)\right|=A_{i}^{ \pm}(n, q) B_{i}^{ \pm}(n, q)$, and $\left\{C_{i, j}^{ \pm}(n, q)\right\}_{j=0}^{N_{i}^{ \pm}(n, q)}$ is the weight distribution of the ternary linear code $C\left(D C_{i}^{ \pm}(n, q)\right)$ given by

$$
\begin{aligned}
& C_{i, j}^{ \pm}(n, q) \\
= & \sum\left(\begin{array}{c}
q^{-1} A_{i}^{ \pm}(n, q)\left(B_{i}^{ \pm}(n, q) \pm 1\right) \\
\nu_{1}, \mu_{1}
\end{array}\right)\left(\begin{array}{c}
q^{-1} A_{i}^{ \pm}(n, q)\left(B_{i}^{ \pm}(n, q) \pm 1\right) \\
\nu_{-1}, \mu_{-1}
\end{array}\right) \\
& \times \prod_{\beta^{2}-1 \neq 0 \text { square }}\left(\begin{array}{c}
q^{-1} A_{i}^{ \pm}(n, q)\left(B_{i}^{ \pm}(n, q) \pm(q+1)\right) \\
\nu_{\beta}, \mu_{\beta}
\end{array}\right) \\
& \times \prod_{\beta^{2}-1} \prod_{\text {nonsquare }}\left(\begin{array}{c}
q^{-1} A_{i}^{ \pm}(n, q)\left(B_{i}^{ \pm}(n, q) \pm(-q+1)\right) \\
\nu_{\beta}, \mu_{\beta}
\end{array}\right),
\end{aligned}
$$

with the sum running over all the sets of nonnegative integers $\left\{\nu_{\beta}\right\}_{\beta \in \mathbb{F}_{q}}$ and $\left\{\mu_{\beta}\right\}_{\beta \in \mathbb{F}_{q}}$ satisfying

$$
\sum_{\beta \in \mathbb{F}_{q}} \nu_{\beta}+\sum_{\beta \in \mathbb{F}_{q}} \mu_{\beta}=j, \quad \text { and } \quad \sum_{\beta \in \mathbb{F}_{q}} \nu_{\beta} \beta=\sum_{\beta \in \mathbb{F}_{q}} \mu_{\beta} \beta .
$$


In addition, $S(h, t)$ is the Stirling number of the second kind defined by

$$
S(h, t)=\frac{1}{t !} \sum_{j=0}^{t}(-1)^{t-j}\left(\begin{array}{l}
t \\
j
\end{array}\right) j^{h} .
$$

(2) With + signs everywhere for \pm signs, we have recursive formulas generating even power moments of Kloosterman sums with square arguments over $\mathbb{F}_{q}$ for each $n \geq 2$ even and all $q$; with - signs everywhere for \pm signs, we have such a formula for each $n \geq 3$ odd and all $q$.

$$
\begin{aligned}
& ( \pm 1)^{h} S K^{2 h} \\
= & -\sum_{l=0}^{h-1}( \pm 1)^{l}\left(\begin{array}{l}
h \\
l
\end{array}\right) B_{2}^{ \pm}(n, q)^{h-l} S K^{2 l} \\
& +q A_{2}^{ \pm}(n, q)^{-h} \sum_{j=0}^{\min \left\{N_{2}^{ \pm}(n, q), h\right\}}(-1)^{j} C_{2, j}^{ \pm}(n, q) \\
& \times \sum_{t=j}^{h} t ! S(h, t) 3^{h-t} 2^{t-h-j-1}\left(\begin{array}{c}
N_{2}^{ \pm}(n, q)-j \\
N_{2}^{ \pm}(n, q)-t
\end{array}\right)(h=1,2, \ldots),
\end{aligned}
$$

where $N_{2}^{ \pm}(n, q)=\left|D C_{2}^{ \pm}(n, q)\right|=A_{2}^{ \pm}(n, q) B_{2}^{ \pm}(n, q)$, and $\left\{C_{2, j}^{ \pm}(n, q)\right\}_{j=0}^{N_{2}^{ \pm}(n, q)}$ is the weight distribution of the ternary linear code $C\left(D C_{2}^{ \pm}(n, q)\right)$ given by

$$
\begin{aligned}
& C_{2, j}^{ \pm}(n, q) \\
= & \sum \prod_{\beta \in \mathbb{F}_{q}}\left(\begin{array}{c}
q^{-1} A_{2}^{ \pm}(n, q)\left(B_{2}^{ \pm}(n, q) \pm\left((q-1)^{2}-q \delta(2, q ; \beta)\right)\right) \\
\nu_{\beta}, \mu_{\beta}
\end{array}\right),
\end{aligned}
$$

with the sum running over all the sets of nonnegative integers $\left\{\nu_{\beta}\right\}_{\beta \in \mathbb{F}_{q}}$ and $\left\{\mu_{\beta}\right\}_{\beta \in \mathbb{F}_{q}}$ satisfying

$$
\sum_{\beta \in \mathbb{F}_{q}} \nu_{\beta}+\sum_{\beta \in \mathbb{F}_{q}} \mu_{\beta}=j, \quad \text { and } \quad \sum_{\beta \in \mathbb{F}_{q}} \nu_{\beta} \beta=\sum_{\beta \in \mathbb{F}_{q}} \mu_{\beta} \beta
$$

and $\delta(2, q ; \beta)=\left|\left\{\left(\alpha_{1}, \alpha_{2}\right) \in \mathbb{F}_{q}^{2} \mid \alpha_{1}+\alpha_{2}+\alpha_{1}^{-1}+\alpha_{2}^{-1}=\beta\right\}\right|$.

(3) With + signs everywhere for \pm signs, we have recursive formulas generating even power moments of Kloosterman sums with square arguments over $\mathbb{F}_{q}$ for each $n \geq 4$ even and all $q$; with - signs everywhere for \pm signs, we have 
such a formula for each $n \geq 3$ odd and all $q$.

$$
\begin{aligned}
& ( \pm 1)^{h} S K^{2 h} \\
= & -\sum_{l=0}^{h-1}( \pm 1)^{l}\left(\begin{array}{l}
h \\
l
\end{array}\right)\left\{B_{4}^{ \pm}(n, q) \pm\left(q^{2}-q\right)\right\}^{h-l} S K^{2 l} \\
& +q A_{4}^{ \pm}(n, q)^{-h} \sum_{j=0}^{\min \left\{N_{4}^{ \pm}(n, q), h\right\}}(-1)^{j} C_{4, j}^{ \pm}(n, q) \\
& \times \sum_{t=j}^{h} t ! S(h, t) 3^{h-t} 2^{t-h-j-1}\left(\begin{array}{c}
N_{4}^{ \pm}(n, q)-j \\
N_{4}^{ \pm}(n, q)-t
\end{array}\right)(h=1,2, \ldots),
\end{aligned}
$$

where $N_{4}^{ \pm}(n, q)=\left|D C_{4}^{ \pm}(n, q)\right|=A_{4}^{ \pm}(n, q) B_{4}^{ \pm}(n, q)$, and $\left\{C_{4, j}^{ \pm}(n, q)\right\}_{j=0}^{N_{4}^{ \pm}(n, q)}$ is the weight distribution of the ternary linear code $C\left(D C_{4}^{ \pm}(n, q)\right)$ given by

$$
\begin{aligned}
& =\sum\left(\begin{array}{c}
q^{-1} A_{4}^{ \pm}(n, q)\left(B_{4}^{ \pm}(n, q) \pm(-1)\left(q \delta(2, q ; \beta)+(q-1)^{3}\right)\right) \\
\nu_{0}, \mu_{0}
\end{array}\right) \\
& \quad \times \prod_{\beta \neq 0}\left(\begin{array}{c}
q^{-1} A_{4}^{ \pm}(n, q)\left(B_{4}^{ \pm}(n, q) \pm(-1)\left(q \delta(2, q ; \beta)-2 q^{2}+3 q-1\right)\right) \\
\nu_{\beta}, \mu_{\beta}
\end{array}\right),
\end{aligned}
$$

with the sum running over all the sets of nonnegative integers $\left\{\nu_{\beta}\right\}_{\beta \in \mathbb{F}_{q}}$ and $\left\{\mu_{\beta}\right\}_{\beta \in \mathbb{F}_{q}}$ satisfying

$$
\sum_{\beta \in \mathbb{F}_{q}} \nu_{\beta}+\sum_{\beta \in \mathbb{F}_{q}} \mu_{\beta}=j, \quad \text { and } \quad \sum_{\beta \in \mathbb{F}_{q}} \nu_{\beta} \beta=\sum_{\beta \in \mathbb{F}_{q}} \mu_{\beta} \beta .
$$

\section{2. $O^{-}(2 n, q)$}

For more details about this section, one is referred to the paper [4] and [5]. Throughout this paper, the following notations will be used:

$$
\begin{aligned}
q & =3^{r}\left(r \in \mathbb{Z}_{>0}\right), \\
\mathbb{F}_{q} & =\text { the finite field with } q \text { elements, } \\
\operatorname{Tr} A & =\text { the trace of } A \text { for a square matrix } A, \\
{ }^{t} B & =\text { the transpose of } B \text { for any matrix } B .
\end{aligned}
$$

The orthogonal group $O^{-}(2 n, q)$ over the field $\mathbb{F}_{q}$ is defined as:

$$
O^{-}(2 n, q)=\left\{w \in G L(2 n, q) \mid{ }^{t} w J w=J\right\},
$$

where

$$
J=\left[\begin{array}{cccc}
0 & 1_{n-1} & 0 & 0 \\
1_{n-1} & 0 & 0 & 0 \\
0 & 0 & 1 & 0 \\
0 & 0 & 0 & -\epsilon
\end{array}\right]
$$


and $\epsilon$ is a fixed element in $\mathbb{F}_{q}^{*} \backslash \mathbb{F}_{q}^{* 2}$, here and throughout this paper.

For convenience, we put

$$
\delta_{\epsilon}=\left[\begin{array}{cc}
1 & 0 \\
0 & -\epsilon
\end{array}\right] .
$$

Then $O^{-}(2 n, q)$ consists of all matrices

$$
\left[\begin{array}{lll}
A & B & e \\
C & D & f \\
g & h & i
\end{array}\right]
$$

$(A, B, C, D:(n-1) \times(n-1), e, f:(n-1) \times 2, g, h: 2 \times(n-1), i: 2 \times 2)$

in $G L(2 n, q)$ satisfying the relations:

$$
\begin{aligned}
& { }^{t} A C+{ }^{t} C A+{ }^{t} g \delta_{\epsilon} g=0, \\
& { }^{t} B D+{ }^{t} D B+{ }^{t} h \delta_{\epsilon} h=0, \\
& { }^{t} e f+{ }^{t} f e+{ }^{t} i \delta_{\epsilon} i=\delta_{\epsilon}, \\
& { }^{t} A D+{ }^{t} C B+{ }^{t} g \delta_{\epsilon} h=1_{n-1}, \\
& { }^{t} A f+{ }^{t} C e+{ }^{t} g \delta_{\epsilon} i=0, \\
& { }^{t} B f+{ }^{t} D e+{ }^{t} h \delta_{\epsilon} i=0 .
\end{aligned}
$$

The special orthogonal group $S O^{-}(2 n, q)$ over the field $\mathbb{F}_{q}$ is defined as:

$$
S O^{-}(2 n, q)=\left\{w \in O^{-}(2 n, q) \mid \operatorname{det} w=1\right\},
$$

which is a subgroup of index 2 in $O^{-}(2 n, q)$.

In particular, we have

$$
\begin{aligned}
O^{-}(2, q) & =\left\{i \in G L(2, q) \mid{ }^{t} i \delta_{\epsilon} i=\delta_{\epsilon}\right\} \\
& =S O^{-}(2, q) \amalg\left[\begin{array}{cc}
1 & 0 \\
0 & -1
\end{array}\right] S O^{-}(2, q),
\end{aligned}
$$

with

$$
\begin{aligned}
S O^{-}(2, q) & =\left\{\left[\begin{array}{cc}
a & b \epsilon \\
b & a
\end{array}\right] \mid a, b \in \mathbb{F}_{q}, a^{2}-b^{2} \epsilon=1\right\} \\
& =\left\{\left[\begin{array}{cc}
a & b \epsilon \\
b & a
\end{array}\right] \mid a+b \epsilon \in \mathbb{F}_{q}(\epsilon) \text { with } N_{\mathbb{F}_{q}(\epsilon) / \mathbb{F}_{q}}(a+b \epsilon)=1\right\} .
\end{aligned}
$$

Let $P(2 n, q)$ be the maximal parabolic subgroup of $O^{-}(2 n, q)$ given by

$$
\begin{aligned}
P & =P(2 n, q) \\
& =\left\{\left[\begin{array}{ccc}
A & 0 & 0 \\
0 & { }^{t} A^{-1} & 0 \\
0 & 0 & i
\end{array}\right]\left[\begin{array}{ccc|c}
1_{n-1} & B & -{ }^{t} h \delta_{\epsilon} \\
0 & 1_{n-1} & 0 \\
0 & h & 1_{2}
\end{array}\right] \mid \begin{array}{c}
A \in G L(n-1, q) \\
i \in O^{-}(2, q) \\
B+B+{ }^{t} h \delta_{\epsilon} h=0
\end{array}\right\},
\end{aligned}
$$


and let $Q=Q(2 n, q)$ be the subgroup of $P(2 n, q)$ of index 2 defined by

$$
\begin{aligned}
Q & =Q(2 n, q) \\
& =\left\{\left[\begin{array}{ccc}
A & 0 & 0 \\
0 & { }^{t} A^{-1} & 0 \\
0 & 0 & i
\end{array}\right]\left[\begin{array}{ccc}
1_{n-1} & B & -{ }^{t} h \delta_{\epsilon} \\
0 & 1_{n-1} & 0 \\
0 & h & 1_{2}
\end{array}\right] \mid \begin{array}{c}
A \in G L(n-1, q) \\
i \in S O^{-}(2, q) \\
{ }^{t} B+B+{ }^{t} h \delta_{\epsilon} h=0
\end{array}\right\} .
\end{aligned}
$$

From (2.2), we see that

$$
P=Q \amalg \rho Q,
$$

with

$$
\rho=\left[\begin{array}{cccc}
1_{n-1} & 0 & 0 & 0 \\
0 & 1_{n-1} & 0 & 0 \\
0 & 0 & 1 & 0 \\
0 & 0 & 0 & -1
\end{array}\right]
$$

Let $\sigma_{r}$ denote the following matrix in $O^{-}(2 n, q)$

$$
\sigma_{r}=\left[\begin{array}{ccccc}
0 & 0 & 1_{r} & 0 & 0 \\
0 & 1_{n-1-r} & 0 & 0 & 0 \\
1_{r} & 0 & 0 & 0 & 0 \\
0 & 0 & 0 & 1_{n-1-r} & 0 \\
0 & 0 & 0 & 0 & 1_{2}
\end{array}\right] \quad(0 \leq r \leq n-1) .
$$

Then the Bruhat decomposition of $O^{-}(2 n, q)$ with respect to $P=P(2 n, q)$ is given by

$$
O^{-}(2 n, q)=\coprod_{r=0}^{n-1} P \sigma_{r} P=\coprod_{r=0}^{n-1} P \sigma_{r} Q
$$

which can further be modified as

$$
\begin{aligned}
O^{-}(2 n, q) & =\coprod_{r=0}^{n-1} P \sigma_{r}\left(B_{r} \backslash Q\right) \\
& =\coprod_{r=0}^{n-1} Q \sigma_{r}\left(B_{r} \backslash Q\right) \amalg \coprod_{r=0}^{n-1}(\rho Q) \sigma_{r}\left(B_{r} \backslash Q\right),
\end{aligned}
$$

with

$$
B_{r}=B_{r}(q)=\left\{w \in Q(2 n, q) \mid \sigma_{r} w \sigma_{r}^{-1} \in P\right\} .
$$

The order of the general linear group $G L(n, q)$ is given by

$$
g_{n}=\prod_{j=0}^{n-1}\left(q^{n}-q^{j}\right)=q^{\left(\begin{array}{c}
n \\
2
\end{array}\right)} \prod_{j=1}^{n}\left(q^{j}-1\right) .
$$


For integers $n, r$ with $0 \leq r \leq n$, the $q$-binomial coefficients are defined as:

$$
\left[\begin{array}{l}
n \\
r
\end{array}\right]_{q}=\prod_{j=0}^{r-1}\left(q^{n-j}-1\right) /\left(q^{r-j}-1\right) .
$$

Then one can show that

$$
\begin{gathered}
|P(2 n, q)|=2(q+1) g_{n-1} q^{(n-1)(n+2) / 2}, \\
\left|B_{r} \backslash Q\right|=\left[\begin{array}{c}
n-1 \\
r
\end{array}\right]_{q} q^{r(r+3) / 2}(0 \leq r \leq n-1)
\end{gathered}
$$

(cf. [4], (3.12), (3.20), (3.21)),

$$
\begin{aligned}
\left|Q(2 n, q) \sigma_{r} Q(2 n, q)\right| & =\left|\rho Q(2 n, q) \sigma_{r} Q(2 n, q)\right| \\
& =\frac{1}{2}\left|P(2 n, q) \sigma_{r} Q(2 n, q)\right| \\
& =\frac{1}{2}|P(2 n, q)|\left|B_{r} \backslash Q(2 n, q)\right| \\
& =(q+1) q^{n^{2}-n} \prod_{j=1}^{n-1}\left(q^{j}-1\right)\left[\begin{array}{c}
n-1 \\
r
\end{array}\right]_{q} q^{\left(\begin{array}{c}
r \\
2
\end{array}\right)} q^{2 r} .
\end{aligned}
$$

Let

$$
\begin{gathered}
D C_{1}^{+}(n, q)=Q(2 n, q) \sigma_{n-1} Q(2 n, q) \text { for } n=2,4,6, \ldots, \\
D C_{2}^{+}(n, q)=Q(2 n, q) \sigma_{n-2} Q(2 n, q) \text { for } n=2,4,6, \ldots, \\
D C_{3}^{+}(n, q)=\rho Q(2 n, q) \sigma_{n-2} Q(2 n, q) \text { for } n=2,4,6, \ldots, \\
D C_{4}^{+}(n, q)=\rho Q(2 n, q) \sigma_{n-3} Q(2 n, q) \text { for } n=4,6,8, \ldots, \\
D C_{1}^{-}(n, q)=Q(2 n, q) \sigma_{n-1} Q(2 n, q) \text { for } n=1,3,5, \ldots, \\
D C_{2}^{-}(n, q)=Q(2 n, q) \sigma_{n-2} Q(2 n, q) \text { for } n=3,5,7 \ldots, \\
D C_{3}^{-}(n, q)=\rho Q(2 n, q) \sigma_{n-2} Q(2 n, q) \text { for } n=3,5,7, \ldots, \\
D C_{4}^{-}(n, q)=\rho Q(2 n, q) \sigma_{n-3} Q(2 n, q) \text { for } n=3,5,7, \ldots
\end{gathered}
$$

Then, from (2.7), we have:

$$
N_{i}^{ \pm}(n, q)=\left|D C_{i}^{ \pm}(n, q)\right|=A_{i}^{ \pm}(n, q) B_{i}^{ \pm}(n, q) \text { for } i=1,2,3,4
$$

(cf. (1.3)-(1.18)).

Unless otherwise stated, from now on, we will agree that anything related to $D C_{1}^{+}(n, q), D C_{2}^{+}(n, q)$ and $D C_{3}^{+}(n, q)$ are defined for $n=2,4,6, \ldots$, anything related to $D C_{4}^{+}(n, q)$ is defined for $n=4,6,8, \ldots$, anything related to $D C_{1}^{-}(n, q)$ is defined for $n=1,3,5, \ldots$, and anything related to $D C_{2}^{-}(n, q)$, $D C_{3}^{-}(n, q)$, and $D C_{4}^{-}(n, q)$ are defined for $n=3,5,7, \ldots$ 


\section{Exponential sums over double cosets of $O^{-}(2 n, q)$}

The following notations will be employed throughout this paper.

$$
\begin{aligned}
& \operatorname{tr}(x)=x+x^{3}+\cdots+x^{3^{r-1}} \text { the trace function } \mathbb{F}_{q} \rightarrow \mathbb{F}_{3}, \\
& \lambda_{0}(x)=e^{2 \pi i x / 3} \text { the canonical additive character of } \mathbb{F}_{3}, \\
& \lambda(x)=e^{2 \pi i \operatorname{tr}(x) / 3} \text { the canonical additive character of } \mathbb{F}_{q} .
\end{aligned}
$$

Then any nontrivial additive character $\psi$ of $\mathbb{F}_{q}$ is given by $\psi(x)=\lambda(a x)$ for a unique $a \in \mathbb{F}_{q}^{*}$.

For any nontrivial additive character $\psi$ of $\mathbb{F}_{q}$ and $a \in \mathbb{F}_{q}^{*}$, the Kloosterman sum $K_{G L(t, q)}(\psi ; a)$ for $G L(t, q)$ is defined as

$$
K_{G L(t, q)}(\psi ; a)=\sum_{w \in G L(t, q)} \psi\left(\operatorname{Tr} w+a \operatorname{Tr} w^{-1}\right) .
$$

Notice that, for $t=1, K_{G L(1, q)}(\psi ; a)$ denotes the Kloosterman sum $K(\psi ; a)$.

In [6], it is shown that $K_{G L(t, q)}(\psi ; a)$ satisfies the following recursive relation: for integers $t \geq 2, a \in \mathbb{F}_{q}^{*}$,

$$
\begin{aligned}
& K_{G L(t, q)}(\psi ; a) \\
= & q^{t-1} K_{G L(t-1, q)}(\psi ; a) K(\psi ; a)+q^{2 t-2}\left(q^{t-1}-1\right) K_{G L(t-2, q)}(\psi ; a),
\end{aligned}
$$

where we understand that $K_{G L(0, q)}(\psi, a)=1$.

Proposition 3.1 ([4]). Let $\psi$ be a nontrivial additive character of $\mathbb{F}_{q}$. For each positive integer $r$, let $\Omega_{r}$ be the set of all $r \times r$ nonsingular symmetric matrices over $F_{q}$. Then, with $\delta_{\epsilon}$ as in (2.1), we have

$$
\begin{aligned}
b_{r}(\psi) & =\sum_{B \in \Omega_{r}} \sum_{h \in \mathbb{F}_{q}^{r \times 2}} \psi\left(\operatorname{Tr} \delta_{\epsilon}^{t} h B h\right) \\
& = \begin{cases}q^{r(r+6) / 4} \prod_{j=1}^{r / 2}\left(q^{2 j-1}-1\right) & \text { for } r \text { even, } \\
-q^{\left(r^{2}+4 r-1\right) / 4} \prod_{j=1}^{(r+1) / 2}\left(q^{2 j-1}-1\right) & \text { for } r \text { odd. }\end{cases}
\end{aligned}
$$

Proposition $3.2([5])$. Let $\psi$ be a nontrivial additive character of $\mathbb{F}_{q}$. Then

$$
\begin{gathered}
\sum_{w \in S O^{-}(2, q)} \psi(\operatorname{Tr} w)=-K(\psi ; 1), \\
\sum_{w \in S O^{-}(2, q)} \psi\left(\operatorname{Tr} \delta_{1} w\right)=q+1, \\
\sum_{i \in O^{-}(2, q)} \psi(\operatorname{Tr} w)=-K(\psi ; 1)+q+1(\mathrm{cf} .(2.2)),
\end{gathered}
$$

where

$$
\delta_{1}=\left[\begin{array}{cc}
1 & 0 \\
0 & -1
\end{array}\right]
$$


Also, from Section 6 of [4], it is shown that Gauss sum for $O^{-}(2 n, q)$, with $\psi$ a nontrivial additive character of $\mathbb{F}_{q}$, is given by:

$$
\begin{aligned}
& \sum_{w \in O^{-}(2 n, q)} \psi(\operatorname{Tr} w) \\
= & \sum_{r=0}^{n-1} \sum_{w \in P \sigma_{r} Q} \psi(\operatorname{Tr} w) \\
= & \sum_{r=0}^{n-1} \sum_{w \in Q \sigma_{r} Q} \psi(\operatorname{Tr} w)+\sum_{r=0}^{n-1} \sum_{w \in \rho Q \sigma_{r} Q} \psi(\operatorname{Tr} w) \quad(\mathrm{cf} .(2.3),(2.4)),
\end{aligned}
$$

with

$$
\begin{aligned}
\sum_{w \in Q \sigma_{r} Q} \psi(\operatorname{Tr} w)= & \left|B_{r} \backslash Q\right| \sum_{w \in Q} \psi\left(\operatorname{Tr} w \sigma_{r}\right) \\
= & q^{(n-1)(n+2) / 2} \sum_{i \in S O^{-}(2, q)} \psi(\operatorname{Tr} i) \\
& \times\left|B_{r} \backslash Q\right| q^{r(n-r-3)} b_{r}(\psi) K_{G L(n-1-r, q)}(\psi ; 1), \\
\sum_{w \in \rho Q \sigma_{r} Q} \psi(\operatorname{Tr} w)= & \left|B_{r} \backslash Q\right| \sum_{w \in Q} \psi\left(\operatorname{Tr} \rho w \sigma_{r}\right) \\
= & q^{(n-1)(n+2) / 2 \sum_{i \in S O^{-}(2, q)} \psi\left(\operatorname{Tr} \delta_{1} i\right)} \\
& \times\left|B_{r} \backslash Q\right| q^{r(n-r-3)} b_{r}(\psi) K_{G L(n-1-r, q)}(\psi ; 1) .
\end{aligned}
$$

Here one uses (2.5) and the fact that $\rho^{-1} w \rho \in Q$ for all $w \in Q$.

Now, we see from (2.6) and Propositions 3.1 and 3.2 that, for each $r$ with $0 \leq r \leq n-1$

$$
\begin{aligned}
& \sum_{w \in Q \sigma_{r} Q} \psi(\operatorname{Tr} w)=q^{(n-1)(n+2) / 2}\left[\begin{array}{c}
n-1 \\
r
\end{array}\right]_{q} K(\psi ; 1) K_{G L(n-1-r, q)}(\psi ; 1) \\
& \times \begin{cases}-q^{r n-\frac{1}{4} r^{2}} \prod_{j=1}^{r / 2}\left(q^{2 j-1}-1\right) & \text { for } r \text { even, } \\
q^{r n-\frac{1}{4}(r+1)^{2}} \prod_{j=1}^{(r+1) / 2}\left(q^{2 j-1}-1\right) & \text { for } r \text { odd },\end{cases} \\
& \sum_{w \in \rho Q \sigma_{r} Q} \psi(\operatorname{Tr} w)=(q+1) q^{(n-1)(n+2) / 2}\left[\begin{array}{c}
n-1 \\
r
\end{array}\right] K_{q L(n-1-r, q)}(\psi ; 1) \\
& \times \begin{cases}q^{r n-\frac{1}{4} r^{2}} \prod_{j=1}^{r / 2}\left(q^{2 j-1}-1\right) & \text { for } r \text { even }, \\
-q^{r n-\frac{1}{4}(r+1)^{2}} \prod_{j=1}^{(r+1) / 2}\left(q^{2 j-1}-1\right) & \text { for } r \text { odd }\end{cases}
\end{aligned}
$$

For our purposes, we need the following special cases of exponential sums in (3.2) and (3.3). We state them separately as a theorem. 
Theorem 3.3. Let $\psi$ be a nontrivial additive character of $\mathbb{F}_{q}$. Then, in the notations of (1.3), (1.5), (1.7), (1.9), (1.11), (1.13), (1.15), and (1.17), we have

$$
\begin{aligned}
\sum_{w \in D C_{i}^{ \pm}(n, q)} \psi(\operatorname{Tr} w) & = \pm A_{i}^{ \pm}(n, q) K(\psi ; 1) \quad \text { for } i=1,3, \\
\sum_{w \in D C_{2}^{ \pm}(n, q)} \psi(\operatorname{Tr} w) & = \pm(-1) A_{2}^{ \pm}(n, q) K(\psi ; 1)^{2}, \\
\sum_{w \in D C_{4}^{ \pm}(n, q)} \psi(\operatorname{Tr} w) & = \pm(-1) q^{-1} A_{4}^{ \pm}(n, q) K_{G L(2, q)}(\psi ; 1) \\
& = \pm(-1) A_{4}^{ \pm}(n, q)\left(K(\psi ; 1)^{2}+q^{2}-q\right)
\end{aligned}
$$

(cf. (2.8)-(2.15),(3.1)).

Corollary 3.4. Let $\lambda$ be the canonical additive character of $\mathbb{F}_{q}$, and let $a \in \mathbb{F}_{q}^{*}$. Then we have

$$
\begin{gathered}
\sum_{w \in D C_{i}^{ \pm}(n, q)} \lambda(a \operatorname{Tr} w)= \pm A_{i}^{ \pm}(n, q) K\left(\lambda ; a^{2}\right) \quad \text { for } i=1,3, \\
\sum_{w \in D C_{2}^{ \pm}(n, q)} \lambda(a \operatorname{Tr} w)= \pm(-1) A_{2}^{ \pm}(n, q) K\left(\lambda ; a^{2}\right)^{2}, \\
\sum_{w \in D C_{4}^{ \pm}(n, q)} \lambda(a \operatorname{Tr} w)= \pm(-1) A_{4}^{ \pm}(n, q)\left(K\left(\lambda ; a^{2}\right)^{2}+q^{2}-q\right) .
\end{gathered}
$$

Proposition $3.5\left([7,(5.3-5))\right.$. Let $\lambda$ be the canonical additive character of $\mathbb{F}_{q}$, $m \in \mathbb{Z}_{\geq 0}, \beta \in \mathbb{F}_{q}$. Then

$$
\sum_{a \in \mathbb{F}_{q}^{*}} \lambda(-a \beta) K\left(\lambda ; a^{2}\right)^{m}=q \delta(m, q ; \beta)-(q-1)^{m},
$$

where, for $m \geq 1$,

(3.8) $\delta(m, q ; \beta)=\left|\left\{\left(\alpha_{1}, \ldots, \alpha_{m}\right) \in\left(\mathbb{F}_{q}^{*}\right)^{m} \mid \alpha_{1}+\alpha_{1}^{-1}+\cdots+\alpha_{m}+\alpha_{m}^{-1}=\beta\right\}\right|$,

and

$$
\delta(0, q ; \beta)= \begin{cases}1, & \text { if } \beta=0, \\ 0, & \text { otherwise } .\end{cases}
$$

Remark 3.6. Here one notes that

$$
\begin{aligned}
\delta(1, q ; \beta)= & \left\{x \in \mathbb{F}_{q} \mid x^{2}-\beta x+1=0\right\} \mid \\
= & \begin{cases}2, & \text { if } \beta^{2}-1 \neq 0 \text { is a square, } \\
1, & \text { if } \beta= \pm 1, \\
0, & \text { if } \beta^{2}-1 \text { is a nonsquare. }\end{cases}
\end{aligned}
$$

In the following lemma, $q$ is not just a power of 3 but a power of any prime. 
Lemma 3.7. For any $\beta$,

$$
\delta(2, q ; \beta) \leq \begin{cases}2 q-4, & \text { if } \operatorname{char} \mathbb{F}_{q} \neq 2, \\ 2 q-3, & \text { if char } \mathbb{F}_{q}=2 .\end{cases}
$$

Proof. Firstly, we show that $\delta(2, q ; \beta) \leq \delta(2, q ; 0)$ for any $\beta$. Observe that

$$
\delta(2, q ; \beta)=\left|\left\{\left(\alpha_{1}, \alpha_{2}\right) \in \mathbb{F}_{q}^{2} \mid \alpha_{1}-\alpha_{2}+\alpha_{1}^{-1}-\alpha_{2}^{-1}=\beta\right\}\right| .
$$

Then, borrowing an idea from [19], we have

$$
\begin{aligned}
\delta(2, q ; \beta) & =q^{-1} \sum_{\alpha \in \mathbb{F}_{q}} \lambda(-\alpha \beta) \sum_{\alpha_{1} \in \mathbb{F}_{q}^{*}} \lambda\left(\alpha\left(\alpha_{1}+\alpha_{1}^{-1}\right)\right) \sum_{\alpha_{2} \in \mathbb{F}_{q}^{*}} \lambda\left(-\alpha\left(\alpha_{2}+\alpha_{2}^{-1}\right)\right) \\
& =q^{-1} \sum_{\alpha \in \mathbb{F}_{q}} \lambda(-\alpha \beta)\left|\sum_{x \in \mathbb{F}_{q}^{*}} \lambda\left(\alpha\left(x+x^{-1}\right)\right)\right|^{2} \\
& \leq q^{-1} \sum_{\alpha \in \mathbb{F}_{q}} \sum_{x \in \mathbb{F}_{q}^{*}}\left|\lambda\left(\alpha\left(x+x^{-1}\right)\right)\right|^{2} \\
& =\delta(2, q ; 0) .
\end{aligned}
$$

Here, for any prime power $q, \lambda$ is the canonical additive character of $\mathbb{F}_{q}$.

Secondly, we show that

$$
\delta(2, q ; 0)= \begin{cases}2 q-4, & \text { if } \operatorname{char} \mathbb{F}_{q} \neq 2, \\ 2 q-3, & \text { if char } \mathbb{F}_{q}=2 .\end{cases}
$$

We see, by multiplying the equation $\alpha_{1}+\alpha_{2}+\alpha_{1}^{-1}+\alpha_{2}^{-1}=0$ by $\alpha_{1} \alpha_{2}$, that

$$
\delta(2, q ; 0)=\left|\left\{\left(\alpha_{1}, \alpha_{2}\right) \in \mathbb{F}_{q}^{2} \mid\left(\alpha_{1} \alpha_{2}+1\right)\left(\alpha_{1}+\alpha_{2}\right)=0\right\}\right|-1,
$$

and

$$
\left\{\left(\alpha_{1}, \alpha_{2}\right) \in \mathbb{F}_{q}^{2} \mid\left(\alpha_{1} \alpha_{2}+1\right)\left(\alpha_{1}+\alpha_{2}\right)=0\right\}=A \cup B,
$$

with

$$
A=\left\{\left(\alpha_{1}, \alpha_{2}\right) \in \mathbb{F}_{q}^{2} \mid \alpha_{1} \alpha_{2}+1=0\right\}, B=\left\{\left(\alpha_{1}, \alpha_{2}\right) \in \mathbb{F}_{q}^{2} \mid \alpha_{1}+\alpha_{2}=0\right\} .
$$

Note here that $|A|=q-1$, and $|B|=q$.

Further, $A \cap B=\{ \pm(1,-1)\}$, so that

$$
|A \cap B|= \begin{cases}2, & \text { if char } \mathbb{F}_{q} \neq 2, \\ 1, & \text { if char } \mathbb{F}_{q}=2 .\end{cases}
$$

From (3.12)-(3.15), we get the result in (3.11).

Remark 3.8. We have shown in [10] that, for char $\mathbb{F}_{q}=2$,

$$
\delta(2, q ; \beta)=\left\{\begin{array}{l}
2 q-3, \quad \text { if } \beta=0, \\
K\left(\lambda ; \beta^{-1}\right)+q-3, \quad \text { if } \beta \neq 0 .
\end{array}\right.
$$


For any integer $r$ with $0 \leq r \leq n-1$, and each $\beta \in \mathbb{F}_{q}$, we let

$$
\begin{aligned}
& N_{Q \sigma_{r} Q}(\beta)=\left|\left\{w \in Q \sigma_{r} Q \mid \operatorname{Tr} w=\beta\right\}\right|, \\
& N_{\rho Q \sigma_{r} Q}(\beta)=\left|\left\{w \in \rho Q \sigma_{r} Q \mid \operatorname{Tr} w=\beta\right\}\right| .
\end{aligned}
$$

Then it is easy to see that

$$
\begin{aligned}
& q N_{Q \sigma_{r} Q}(\beta)=\left|Q \sigma_{r} Q\right|+\sum_{a \in \mathbb{F}_{q}^{*}} \lambda(-a \beta) \sum_{w \in Q \sigma_{r} Q} \lambda(a \operatorname{Tr} w), \\
& q N_{\rho Q \sigma_{r} Q}(\beta)=\left|\rho Q \sigma_{r} Q\right|+\sum_{a \in \mathbb{F}_{q}^{*}} \lambda(-a \beta) \sum_{w \in \rho Q \sigma_{r} Q} \lambda(a \operatorname{Tr} w) .
\end{aligned}
$$

Now, from (2.8)-(2.16) and (3.4)-(3.7), we have the following result.

Proposition 3.9. (1) For $i=1,3$,

$$
\begin{aligned}
N_{D C_{i}^{ \pm}(n, q)}(\beta)= & q^{-1} A_{i}^{ \pm}(n, q) B_{i}^{ \pm}(n, q) \pm q^{-1} A_{i}^{ \pm}(n, q)(q \delta(1, q ; \beta)-q+1) \\
= & q^{-1} A_{i}^{ \pm}(n, q) B_{i}^{ \pm}(n, q) \pm q^{-1} A_{i}^{ \pm}(n, q) \\
& \times \begin{cases}q+1, & \text { if } \beta^{2}-1 \neq 0 \text { is a square, } \\
1, & \text { if } \beta= \pm 1, \\
-q+1, & \text { if } \beta^{2}-1 \text { is a nonsquare. }\end{cases}
\end{aligned}
$$

$$
N_{D C_{2}^{ \pm}(n, q)}(\beta)
$$

$$
=q^{-1} A_{2}^{ \pm}(n, q) B_{2}^{ \pm}(n, q) \pm(-1) q^{-1} A_{2}^{ \pm}(n, q)\left\{q \delta(2, q ; \beta)-(q-1)^{2}\right\} .
$$

$$
N_{D C_{4}^{ \pm}(n, q)}(\beta)=q^{-1} A_{4}^{ \pm}(n, q) B_{4}^{ \pm}(n, q) \pm(-1) q^{-1} A_{4}^{ \pm}(n, q)
$$

$$
\times \begin{cases}q \delta(2, q ; \beta)-2 q^{2}+3 q-1, & \text { if } \beta \neq 0, \\ q \delta(2, q ; \beta)+q^{3}-3 q^{2}+3 q-1, & \text { if } \beta=0\end{cases}
$$

Here $\delta(2, q ; \beta)=\left|\left\{\left(\alpha_{1}, \alpha_{2}\right) \in\left(\mathbb{F}_{q}^{*}\right)^{2} \mid \alpha_{1}+\alpha_{1}^{-1}+\alpha_{2}+\alpha_{2}^{-1}=\beta\right\}\right|$.

Corollary 3.10. (1) For all even $n \geq 2$ and all $q, N_{D C_{i}^{+}(n, q)}(\beta)>0$ for all $\beta$ and $i=1,2$.

(2) For all even $n \geq 4$ and all $q, N_{D C_{3}^{+}(n, q)}(\beta)>0$ for all $\beta$; for $n=2$ and all $q$,

$$
\begin{aligned}
N_{D C_{3}^{+}(2, q)}(\beta) & =q^{2}(q+1) \delta(1, q ; \beta) \\
& =\left\{\begin{array}{l}
2 q^{3}+2 q^{2}, \quad \text { if } \beta^{2}-1 \neq 0 \text { is a square } \\
q^{3}+q^{2}, \quad \text { if } \beta= \pm 1 \\
0, \quad \text { if } \beta^{2}-1 \text { is a nonsquare. }
\end{array}\right.
\end{aligned}
$$

(3) For all even $n \geq 4$ and all $q, N_{D C_{4}^{+}(n, q)}(\beta)>0$ for all $\beta$. 
(4) For all odd $n \geq 3$ and all $q, N_{D C_{1}^{-}(n, q)}(\beta)>0$ for all $\beta$; for $n=1$ and all $q$,

$$
\begin{aligned}
N_{D C_{1}^{-}(1, q)}(\beta)= & 2-\delta(1, q ; \beta) \\
& = \begin{cases}0, & \text { if } \beta^{2}-1 \neq 0 \text { is a square, } \\
1, & \text { if } \beta= \pm 1, \\
2, & \text { if } \beta^{2}-1 \text { is a nonsquare. }\end{cases}
\end{aligned}
$$

(5) For all odd $n \geq 3$ and all $q, N_{D C_{i}^{-}(n, q)}(\beta)>0$ for all $\beta$ and $i=2,3,4$.

Proof. It is tedious to check all the assertions in the statements. The details are left to the reader, except that we make a comment on the case of (1) with $i=2$. We see that $N_{D C_{2}^{+}(n, q)}(\beta)>0$ for all $n \geq 4$ even and all $q$. In addition,

$$
N_{D C_{2}^{+}(2, q)}(\beta)=q^{2}(2 q-2-\delta(2, q ; \beta))>0, \text { in view of }(3.10) \text {. }
$$

\section{Construction of codes}

We will construct eight infinite families of ternary linear codes $C\left(D C_{1}^{+}(n, q)\right)$ of length $\left.N_{1}^{+}(n, q)\right), C\left(D C_{2}^{+}(n, q)\right)$ of length $N_{2}^{+}(n, q), C\left(D C_{3}^{+}(n, q)\right)$ of length $N_{3}^{+}(n, q)$ for $n=2,4,6, \ldots$ and all $q ; C\left(D C_{4}^{+}(n, q)\right)$ of length $N_{4}^{+}(n, q)$ for $n=4,6,8, \ldots$ and all $q ; C\left(D C_{1}^{-}(n, q)\right)$ of length $N_{1}^{-}(n, q)$ for $n=1,3,5, \ldots$ and all $q ; C\left(D C_{2}^{-}(n, q)\right)$ of length $N_{2}^{-}(n, q), C\left(D C_{3}^{-}(n, q)\right)$ of length $N_{3}^{-}(n, q)$, $C\left(D C_{4}^{-}(n, q)\right)$ of length $N_{4}^{-}(n, q)$ for $n=3,5,7, \ldots$ and all $q$, respectively associated with the double cosets $D C_{1}^{+}(n, q), D C_{2}^{+}(n, q), D C_{3}^{+}(n, q), D C_{4}^{+}(n, q)$, $D C_{1}^{-}(n, q), D C_{2}^{-}(n, q), D C_{3}^{-}(n, q), D C_{4}^{-}(n, q)$ (cf. (2.8)-(2.15)). Let $g_{1}, g_{2}, \ldots$, $g_{N_{i}^{ \pm}(n, q)}$ be some fixed orderings of the elements in $D C_{i}^{ \pm}(n, q)$ for $i=1,2,3,4$, by abuse of notations. Then we put

$$
v_{i}^{ \pm}(n, q)=\left(\operatorname{Tr} g_{1}, \operatorname{Tr} g_{2}, \ldots, \operatorname{Tr} g_{N_{i}^{ \pm}(n, q)}\right) \in \mathbb{F}_{q}^{N_{i}^{ \pm}(n, q)} \text { for } i=1,2,3,4 .
$$

The ternary codes $C\left(D C_{1}^{+}(n, q)\right), C\left(D C_{2}^{+}(n, q)\right), C\left(D C_{3}^{+}(n, q)\right), C\left(D C_{4}^{+}(n, q)\right)$, $C\left(D C_{1}^{-}(n, q)\right), C\left(D C_{2}^{-}(n, q)\right), C\left(D C_{3}^{-}(n, q)\right)$, and $C\left(D C_{4}^{-}(n, q)\right)$ are defined as:

$$
C\left(D C_{i}^{ \pm}(n, q)\right)=\left\{u \in \mathbb{F}_{3}^{N_{i}^{ \pm}(n, q)} \mid u \cdot v_{i}^{ \pm}(n, q)=0\right\} \text { for } i=1,2,3,4,
$$

where the dot denotes respectively the usual inner product in $\mathbb{F}_{q}^{N_{i}^{ \pm}(n, q)}$ for $i=1,2,3,4$.

The following theorem of Delsarte is well-known.

Theorem 4.1 ([15]). Let $B$ be a linear code over $\mathbb{F}_{q}$. Then

$$
\left(\left.B\right|_{\mathbb{F}_{3}}\right)^{\perp}=\operatorname{tr}\left(B^{\perp}\right) .
$$


In view of this theorem, the respective duals of the codes in (4.1) are given by:

$$
\begin{aligned}
& C\left(D C_{i}^{ \pm}(n, q)\right)^{\perp} \\
= & \left\{c_{i}^{ \pm}(a)=c_{i}^{ \pm}(a ; n, q)=\left(\operatorname{tr}\left(a \operatorname{Tr} g_{1}\right), \ldots, \operatorname{tr}\left(a \operatorname{Tr} g_{N_{i}^{ \pm}(n, q)}\right)\right) \mid a \in \mathbb{F}_{q}\right\} \\
& \text { for } i=1,2,3,4 .
\end{aligned}
$$

Lemma 4.2. Let $\delta(m, q ; \beta)$ be as in $(3.8)$, and let $a \in \mathbb{F}_{q}^{*}$. Then we have

$$
\sum_{\beta \in \mathbb{F}_{q}} \delta(m, q ; \beta) \lambda(a \beta)=K\left(\lambda ; a^{2}\right)^{m} .
$$

Proof. The LHS of (4.3) is equal to

$$
\begin{aligned}
& \sum_{\beta \in \mathbb{F}_{q}}\left(q^{-1} \sum_{x_{1}, \cdots, x_{m} \in \mathbb{F}_{q}^{*}} \sum_{\alpha \in \mathbb{F}_{q}} \lambda\left(\alpha\left(x_{1}+\cdots+x_{m}+x_{1}^{-1}+\cdots+x_{m}^{-1}-\beta\right)\right)\right) \lambda(a \beta) \\
= & q^{-1} \sum_{x_{1}, \ldots, x_{m} \in \mathbb{F}_{q}^{*}} \sum_{\alpha \in \mathbb{F}_{q}} \lambda\left(\alpha\left(x_{1}+\cdots+x_{m}+x_{1}^{-1}+\cdots+x_{m}^{-1}\right)\right) \sum_{\beta \in \mathbb{F}_{q}} \lambda(\beta(a-\alpha)) \\
= & \sum_{x_{1}, \ldots, x_{m} \in \mathbb{F}_{q}^{*}} \lambda\left(a\left(x_{1}+\cdots+x_{m}+x_{1}^{-1}+\cdots+x_{m}^{-1}\right)\right) \\
= & \sum_{x_{1}, \ldots, x_{m} \in \mathbb{F}_{q}^{*}} \lambda\left(x_{1}+\cdots+x_{m}+a^{2} x_{1}^{-1}+\cdots+a^{2} x_{m}^{-1}\right) \\
= & K\left(\lambda ; a^{2}\right)^{m} .
\end{aligned}
$$

Theorem 4.3. (1) The map $\mathbb{F}_{q} \rightarrow C\left(D C_{i}^{+}(n, q)\right)^{\perp}\left(a \mapsto c_{i}^{+}(a)\right)($ for $i=1,2,3)$ is an $\mathbb{F}_{3}$-linear isomorphism for $n \geq 2$ even and all $q$.

(2) The map $\mathbb{F}_{q} \rightarrow C\left(D C_{4}^{+}(n, q)\right)^{\perp}\left(a \mapsto c_{4}^{+}(a)\right)$ is an $\mathbb{F}_{3}$-linear isomorphism for $n \geq 4$ even and all $q$.

(3) The map $\mathbb{F}_{q} \rightarrow C\left(D C_{1}^{-}(n, q)\right)^{\perp}\left(a \mapsto c_{1}^{-}(a)\right)$ is an $\mathbb{F}_{3}$-linear isomorphism for $n \geq 1$ odd and all $q$.

(4) The map $\mathbb{F}_{q} \rightarrow C\left(D C_{i}^{-}(n, q)\right)^{\perp}\left(a \mapsto c_{i}^{-}(a)\right)($ for $i=2,3,4)$ is an $\mathbb{F}_{3^{-}}$ linear isomorphism for $n \geq 3$ odd and all $q$.

Proof. All maps are clearly $\mathbb{F}_{3}$-linear and surjective. Let $a$ be in the kernel of map $\mathbb{F}_{q} \rightarrow C\left(D C_{1}^{+}(n, q)\right)^{\perp}\left(a \mapsto c_{1}^{+}(a)\right)$. Then $\operatorname{tr}(a \operatorname{Tr} g)=0$ for all $g \in$ $D C_{1}^{+}(n, q)$. Since, by Corollary $3.10(1)$, $\operatorname{Tr}: D C_{1}^{+}(n, q) \rightarrow \mathbb{F}_{q}$ is surjective, and hence $\operatorname{tr}(a \alpha)=0$ for all $\alpha \in \mathbb{F}_{q}$. This implies that $a=0$, since otherwise $\operatorname{tr}: \mathbb{F}_{q} \rightarrow \mathbb{F}_{3}$ would be the zero map. This shows $i=1$ case of (1). All the other assertions can be handled in the same way, except for $i=3$ and $n=2$ case of (1) and $n=1$ case of (3).

Assume first that we are in the $i=3$ and $n=2$ case of (1). Let $a$ be in the kernel of the map $\mathbb{F}_{q} \rightarrow C\left(D C_{3}^{+}(2, q)\right)^{\perp}\left(a \mapsto c_{3}^{+}(a)\right)$. Then $\operatorname{tr}(a \operatorname{Tr} g)=0$ for all 
$g \in D C_{3}^{+}(2, q)$. Suppose that $a \neq 0$. Then we would have

$$
\begin{aligned}
q^{2}\left(q^{2}-1\right)=\left|D C_{3}^{+}(2, q)\right| & =\sum_{g \in D C_{3}^{+}(2, q)} e^{2 \pi i \operatorname{tr}(a \operatorname{Tr} g) / 3} \\
& =\sum_{\beta \in \mathbb{F}_{q}} N_{D C_{3}^{+}(2, q)}(\beta) \lambda(a \beta) \\
& =q^{2}(q+1) \sum_{\beta \in \mathbb{F}_{q}} \delta(1, q ; \beta) \lambda(a \beta)(\mathrm{cf.} \text { (3.19)) } \\
& =q^{2}(q+1) K\left(\lambda ; a^{2}\right)(\mathrm{cf.}(4.3)) .
\end{aligned}
$$

So, using Weil bound in (1.1), we would get

$$
q-1=K\left(\lambda ; a^{2}\right) \leq 2 \sqrt{q} .
$$

For $q \geq 9$, this is impossible, since $x-1>2 \sqrt{x}$ for $x \geq 9$. So the map $\mathbb{F}_{q} \rightarrow C\left(D C_{3}^{+}(2, q)\right)^{\perp}\left(a \mapsto c_{3}^{+}(a)\right)$ is an $\mathbb{F}_{3}$-linear isomorphism if $q \geq 9$. This is also true for $q=3$. Indeed, if $a$ is in the kernel of the map, then $a \operatorname{Tr} g=0$ for all $g \in D C_{3}^{+}(2,3)$. Here $\operatorname{Tr} g=1$ for a half of elements $g \in D C_{3}^{+}(2,3)$, and $\operatorname{Tr} g=-1$ for the other half of elements $g \in D C_{3}^{+}(2,3)$ (cf. (3.19)). So $a=0$. Assume next that we are in the $n=1$ case of (3). Let $a$ be in the kernel of the map $\mathbb{F}_{q} \rightarrow C\left(D C_{1}^{-}(1, q)\right)^{\perp}\left(a \mapsto c_{1}^{-}(a)\right)$. Then $\operatorname{tr}(a \operatorname{Tr} g)=0$ for all $g \in D C_{1}^{-}(1, q)$. Assume that $a \neq 0$. Then, again using Weil bound, we would have

$$
\begin{aligned}
q+1=\left|D C_{1}^{-}(1, q)\right| & =\sum_{g \in D C_{1}^{-}(1, q)} e^{2 \pi i \operatorname{tr}(a \operatorname{Tr} g) / 3} \\
& =\sum_{\beta \in \mathbb{F}_{q}} N_{D C_{1}^{-}(1, q)}(\beta) \lambda(a \beta) \\
& =\sum_{\beta \in \mathbb{F}_{q}}(2-\delta(1, q ; \beta)) \lambda(a \beta)(\mathrm{cf.}(3.20)) \\
& =-\sum_{\beta \in \mathbb{F}_{q}} \delta(1, q ; \beta) \lambda(a \beta)(4.3) \\
& =-K\left(\lambda ; a^{2}\right) \\
& \leq 2 \sqrt{q} .
\end{aligned}
$$

So we would get $q=1$, which is impossible.

\section{Recursive formulas for power moments of Kloosterman sums}

Here we will be able to find, via Pless power moment identity, infinite families of recursive formulas generating power moments of Kloosterman sums with square arguments and even power moments of those in terms of the frequencies of weights in $C\left(D C_{i}^{ \pm}(n, q)\right)$ for $i=1,3$ and in $C\left(D C_{i}^{ \pm}(n, q)\right)$ for $i=2,4$, respectively. 
Theorem 5.1 (Pless power moment identity, [15]). Let $B$ be a q-ary $[n, k]$ code, and let $B_{i}$ (resp. $\left.B_{i}^{\perp}\right)$ denote the number of codewords of weight $i$ in $B$ (resp. in $\left.B^{\perp}\right)$. Then, for $h=0,1,2, \ldots$,

$$
\sum_{j=0}^{n} j^{h} B_{j}=\sum_{j=0}^{\min \{n, h\}}(-1)^{j} B_{j}^{\perp} \sum_{t=j}^{h} t ! S(h, t) q^{k-t}(q-1)^{t-j}\left(\begin{array}{c}
n-j \\
n-t
\end{array}\right),
$$

where $S(h, t)$ is the Stirling number of the second kind defined in (1.21).

Lemma 5.2. Let $c_{i}^{ \pm}(a)=\left(\operatorname{tr}\left(a \operatorname{Tr} g_{1}\right), \ldots, \operatorname{tr}\left(a \operatorname{Tr} g_{N_{i}^{ \pm}(n, q)}\right)\right) \in C\left(D C_{i}^{ \pm}(n, q)\right)^{\perp}$ for $a \in \mathbb{F}_{q}^{*}$, and $i=1,2,3,4$. Then their Hamming weights are expressed as follows:

(1) $\quad w\left(c_{i}^{ \pm}(a)\right)$

$$
=\frac{2}{3} A_{i}^{ \pm}(n, q)\left\{B_{i}^{ \pm}(n, q) \pm(-1) K\left(\lambda ; a^{2}\right)\right\} \text { for } i=1,3 \text {, }
$$

(2) $\quad w\left(c_{2}^{ \pm}(a)\right)$

$$
=\frac{2}{3} A_{2}^{ \pm}(n, q)\left\{B_{2}^{ \pm}(n, q) \pm K\left(\lambda ; a^{2}\right)^{2}\right\}
$$

$$
\begin{aligned}
& w\left(c_{4}^{ \pm}(a)\right) \\
= & \frac{2}{3} A_{4}^{ \pm}(n, q)\left\{B_{4}^{ \pm}(n, q) \pm\left(q^{2}-q+K\left(\lambda ; a^{2}\right)^{2}\right)\right\}(c f .(1.3)-(1.18)) .
\end{aligned}
$$

Proof.

$$
\begin{aligned}
w\left(c_{i}^{ \pm}(a)\right) & =\sum_{j=1}^{N_{i}^{ \pm}(n, q)}\left(1-\frac{1}{3} \sum_{\alpha \in \mathbb{F}_{3}} \lambda_{0}\left(\alpha \operatorname{tr}\left(a \operatorname{Tr} g_{j}\right)\right)\right) \\
& =N_{i}^{ \pm}(n, q)-\frac{1}{3} \sum_{\alpha \in \mathbb{F}_{3}} \sum_{w \in D C_{i}^{ \pm}(n, q)} \lambda(\alpha a \operatorname{Tr} w) \\
& =\frac{2}{3} N_{i}^{ \pm}(n, q)-\frac{1}{3} \sum_{\alpha \in \mathbb{F}_{3}^{*}} \sum_{w \in D C_{i}^{ \pm}(n, q)} \lambda(\alpha a \operatorname{Tr} w) \quad \text { for } i=1,2,3,4 .
\end{aligned}
$$

Our results now follow from (2.16) and (3.4)-(3.6).

Let $u=\left(u_{1}, \ldots, u_{N_{i}^{ \pm}(n, q)}\right) \in \mathbb{F}_{3}^{N_{i}^{ \pm}(n, q)}$ for $i=1,2,3,4$, with $\nu_{\beta} 1$ 's and $\mu_{\beta}$ 2 's in the coordinate places where $\operatorname{Tr}\left(g_{j}\right)=\beta$ for each $\beta \in \mathbb{F}_{q}$. Then, from the definition of the codes $C\left(D C_{i}^{ \pm}(n, q)\right)(\mathrm{cf} .(4.1))$, we see that $u$ is a codeword with weight $j$ if and only if $\sum_{\beta \in \mathbb{F}_{q}} \nu_{\beta}+\sum_{\beta \in \mathbb{F}_{q}} \mu_{\beta}=j$ and $\sum_{\beta \in \mathbb{F}_{q}} \nu_{\beta} \beta=$ $\sum_{\beta \in \mathbb{F}_{q}} \mu_{\beta} \beta$ (an identity in $\mathbb{F}_{q}$ ). As there are $\prod_{\beta \in \mathbb{F}_{q}}\left(\begin{array}{c}N_{D C_{i}^{ \pm}(n, q)}^{ \pm} \\ \nu_{\beta}, \mu_{\beta}\end{array}\right)$ many such codewords with weight $j$, we obtain the following result. 
Proposition 5.3. Let $\left\{C_{i, j}^{ \pm}(n, q)\right\}_{j=0}^{N_{i}^{ \pm}(n, q)}$ be the weight distribution of

$$
C\left(D C_{i}^{ \pm}(n, q)\right)
$$

for $i=1,2,3,4$. Then we have

$$
\begin{aligned}
& C_{i, j}^{ \pm}(n, q) \\
= & \sum \prod_{\beta \in \mathbb{F}_{q}}\left(\begin{array}{c}
N_{D C_{i}^{ \pm}(n, q)}(\beta) \\
\nu_{\beta}, \mu_{\beta}
\end{array}\right) \text { for } 0 \leq j \leq N_{i}^{ \pm}(n, q) \text { and } i=1,2,3,4,
\end{aligned}
$$

where the sum is over all the sets of nonnegative integers $\left\{\nu_{\beta}\right\}_{\beta \in \mathbb{F}_{q}}$ and $\left\{\mu_{\beta}\right\}_{\beta \in \mathbb{F}_{q}}$ satisfying

$$
\sum_{\beta \in \mathbb{F}_{q}} \nu_{\beta}+\sum_{\beta \in \mathbb{F}_{q}} \mu_{\beta}=j, \quad \text { and } \quad \sum_{\beta \in \mathbb{F}_{q}} \nu_{\beta} \beta=\sum_{\beta \in \mathbb{F}_{q}} \mu_{\beta} \beta .
$$

The formulas appearing in the next theorem and stated in (1.20), (1.23), and (1.25) follow by applying the formula in (5.5) to each $C\left(D C_{i}^{ \pm}(n, q)\right)$, using the explicit values of $N_{D C_{i}^{ \pm}(n, q)}(\beta)$ in (3.16)-(3.18).

Theorem 5.4. Let $\left\{C_{i, j}^{ \pm}(n, q)\right\}_{j=0}^{N_{i}^{ \pm}(n, q)}$ be the weight distribution of

$$
C\left(D C_{i}^{ \pm}(n, q)\right)
$$

for $i=1,2,3,4$. Then we have

(1) For $i=1,3$, and $j=0, \ldots, N_{i}^{ \pm}(n, q)$,

$$
\begin{aligned}
C_{i, j}^{ \pm}(n, q)= & \sum\left(\begin{array}{c}
q^{-1} A_{i}^{ \pm}(n, q)\left(B_{i}^{ \pm}(n, q) \pm 1\right) \\
\nu_{1}, \mu_{1}
\end{array}\right)\left(\begin{array}{c}
q^{-1} A_{i}^{ \pm}(n, q)\left(B_{i}^{ \pm}(n, q) \pm 1\right) \\
\nu_{-1}, \mu_{-1}
\end{array}\right) \\
& \times \prod_{\beta^{2}-1 \neq 0 \text { square }}\left(\begin{array}{c}
q^{-1} A_{i}^{ \pm}(n, q)\left(B_{i}^{ \pm}(n, q) \pm(q+1)\right) \\
\nu_{\beta}, \mu_{\beta}
\end{array}\right) \\
& \times \prod_{\beta^{2}-1} \prod_{\text {nonsquare }}\left(\begin{array}{c}
q^{-1} A_{i}^{ \pm}(n, q)\left(B_{i}^{ \pm}(n, q) \pm(-q+1)\right) \\
\nu_{\beta}, \mu_{\beta}
\end{array}\right),
\end{aligned}
$$

where the sum is over all the sets of nonnegative integers $\left\{\nu_{\beta}\right\}_{\beta \in \mathbb{F}_{q}}$ and $\left\{\mu_{\beta}\right\}_{\beta \in \mathbb{F}_{q}}$ satisfying

$$
\sum_{\beta \in \mathbb{F}_{q}} \nu_{\beta}+\sum_{\beta \in \mathbb{F}_{q}} \mu_{\beta}=j, \quad \text { and } \quad \sum_{\beta \in \mathbb{F}_{q}} \nu_{\beta} \beta=\sum_{\beta \in \mathbb{F}_{q}} \mu_{\beta} \beta
$$

(2) For $j=0, \ldots, N_{2}^{ \pm}(n, q)$,

$$
C_{2, j}^{ \pm}(n, q)=\sum \prod_{\beta \in \mathbb{F}_{q}}\left(\begin{array}{c}
q^{-1} A_{2}^{ \pm}(n, q)\left(B_{2}^{ \pm}(n, q) \pm\left((q-1)^{2}-q \delta(2, q ; \beta)\right)\right) \\
\nu_{\beta}, \mu_{\beta}
\end{array}\right),
$$


where the sum is over all the sets of nonnegative integers $\left\{\nu_{\beta}\right\}_{\beta \in \mathbb{F}_{q}}$ and $\left\{\mu_{\beta}\right\}_{\beta \in \mathbb{F}_{q}}$ satisfying

$$
\sum_{\beta \in \mathbb{F}_{q}} \nu_{\beta}+\sum_{\beta \in \mathbb{F}_{q}} \mu_{\beta}=j, \quad \text { and } \quad \sum_{\beta \in \mathbb{F}_{q}} \nu_{\beta} \beta=\sum_{\beta \in \mathbb{F}_{q}} \mu_{\beta} \beta .
$$

(3) For $j=0, \ldots, N_{4}^{ \pm}(n, q)$,

$$
\begin{aligned}
C_{4, j}^{ \pm}(n, q)= & \sum\left(\begin{array}{c}
q^{-1} A_{4}^{ \pm}(n, q)\left(B_{4}^{ \pm}(n, q) \pm(-1)\left(q \delta(2, q ; \beta)+(q-1)^{3}\right)\right) \\
\nu_{0}, \mu_{0}
\end{array}\right) \\
& \times \prod_{\beta \neq 0}\left(\begin{array}{c}
q^{-1} A_{4}^{ \pm}(n, q)\left(B_{4}^{ \pm}(n, q) \pm(-1)\left(q \delta(2, q ; \beta)-2 q^{2}+3 q-1\right)\right) \\
\nu_{\beta}, \mu_{\beta}
\end{array}\right),
\end{aligned}
$$

where the sum is over all the sets of nonnegative integers $\left\{\nu_{\beta}\right\}_{\beta \in \mathbb{F}_{q}}$ and $\left\{\mu_{\beta}\right\}_{\beta \in \mathbb{F}_{q}}$ satisfying

$$
\sum_{\beta \in \mathbb{F}_{q}} \nu_{\beta}+\sum_{\beta \in \mathbb{F}_{q}} \mu_{\beta}=j, \quad \text { and } \quad \sum_{\beta \in \mathbb{F}_{q}} \nu_{\beta} \beta=\sum_{\beta \in \mathbb{F}_{q}} \mu_{\beta} \beta .
$$

Now, we apply the Pless power moment identity in (5.1) to $C\left(D C_{i}^{ \pm}(n, q)\right)$ for $i=1,2,3,4$, in order to get the results in Theorem 1 (cf. (1.19), (1.20), (1.22)-(1.25)) about recursive formulas.

The left hand side of that identity in (5.1) is equal to

$$
\sum_{a \in \mathbb{F}_{q}^{*}} w\left(c_{i}^{ \pm}(a)\right)^{h}
$$

with $w\left(c_{i}^{ \pm}(a)\right)$ given by (5.2)-(5.4). We have, for $i=1,3$,

$$
\begin{aligned}
\sum_{a \in \mathbb{F}_{q}^{*}} w\left(c_{i}^{ \pm}(a)\right)^{h} & =\left(\frac{2}{3}\right)^{h} A_{i}^{ \pm}(n, q)^{h} \sum_{a \in \mathbb{F}_{q}^{*}}\left\{B_{i}^{ \pm}(n, q) \pm(-1) K\left(\lambda ; a^{2}\right)\right\}^{h} \\
& =2\left(\frac{2}{3}\right)^{h} A_{i}^{ \pm}(n, q)^{h} \sum_{l=0}^{h}( \pm(-1))^{l}\left(\begin{array}{c}
h \\
l
\end{array}\right) B_{i}^{ \pm}(n, q)^{h-l} S K^{l} .
\end{aligned}
$$

Similarly, we have

$$
\begin{aligned}
\sum_{a \in \mathbb{F}_{q}^{*}} w\left(c_{2}^{ \pm}(a)\right)^{h}=2\left(\frac{2}{3}\right)^{h} A_{2}^{ \pm}(n, q)^{h} \sum_{l=0}^{h}(( \pm 1))^{l}\left(\begin{array}{c}
h \\
l
\end{array}\right) B_{2}^{ \pm}(n, q)^{h-l} S K^{2 l}, \\
\sum_{a \in \mathbb{F}_{q}^{*}} w\left(c_{4}^{ \pm}(a)\right)^{h} \\
=2\left(\frac{2}{3}\right)^{h} A_{4}^{ \pm}(n, q)^{h} \sum_{l=0}^{h}( \pm 1)^{l}\left(\begin{array}{c}
h \\
l
\end{array}\right)\left\{B_{4}^{ \pm}(n, q) \pm\left(q^{2}-q\right)\right\}^{h-l} S K^{2 l} .
\end{aligned}
$$

Here one has to separate the term corresponding to $l=h$ in (5.6)-(5.8), and notes $\operatorname{dim}_{\mathbb{F}_{3}} C\left(D C_{i}^{ \pm}(n, q)\right)^{\perp}=r$ 
Acknowledgment. The author would like to thank the referee for his or her helpful comments on this paper.

\section{References}

[1] R. J. Evans, Seventh power moments of Kloosterman sums, Israel J. Math. 175 (2010), 349-362.

[2] G. van der Geer, R. Schoof, and M. van der Vlugt, Weight formulas for ternary Melas codes, Math. Comp. 58 (1992), no. 198, 781-792.

[3] K. Hulek, J. Spandaw, B. van Geemen, and D. van Straten, The modularity of the Barth-Nieto quintic and its relatives, Adv. Geom. 1 (2001), no. 3, 263-289.

[4] D. S. Kim, Gauss sums for $\mathrm{O}^{-}(2 n, q)$, Acta Arith. 80 (1997), no. 4, 343-365.

[5] _ Exponential sums for $\mathrm{O}^{-}(2 n, q)$ and their applications, Acta Arith. 97 (2001), no. $1,67-86$.

[6] _ Gauss sums for symplectic groups over a finite field, Monatsh. Math. 126 (1998), no. 1, 55-71.

[7] _ Exponential sums for symplectic groups and their applications, Acta Arith. 88 (1999), no. 2, 155-171.

[8] _ Infinite families of recursive formulas generating power moments of ternary Kloosterman sums with square arguments arising from symplectic groups, Adv. Math. Commun. 3 (2009), no. 2, 167-178.

[9] _ Ternary codes associated with $\mathrm{O}^{-}(2 n, q)$ and power moments of Kloosterman sums with square arguments, submitted.

[10] _ Recursive formulas generating power moments of multi-dimensional Kloosterman sums and m-multiple power moments of Kloosterman sums, submitted.

[11] D. S. Kim and J. H. Kim, Ternary codes associated with symplectic groups and power moments of Kloosterman sums with square arguments, submitted.

[12] H. D. Kloosterman, On the representation of numbers in the form $a x^{2}+b y^{2}+c z^{2}+d t^{2}$, Acta Math. 49 (1927), no. 3-4, 407-464.

[13] R. Lidl and H. Niederreiter, Finite Fields, Encyclopedia Math. Appl. 20, Cambridge University Pless, Cambridge, 1987.

[14] R. Livné, Motivic orthogonal two-dimensional representations of $\operatorname{Gal}(\bar{Q} / \mathbf{Q})$, Israel J. Math. 92 (1995), no. 1-3, 149-156.

[15] F. J. MacWilliams and N. J. A. Sloane, The Theory of Error Correcting Codes, NorthHolland, Amsterdam, 1998

[16] M. Moisio, On the moments of Kloosterman sums and fibre products of Kloosterman curves, Finite Fields Appl. 14 (2008), no. 2, 515-531.

[17] C. Peters, J. Top, and M. van der Vlugt, The Hasse zeta function of a K3 surface related to the number of words of weight 5 in the Melas codes, J. Reine Angew. Math. 432 (1992), 151-176.

[18] H. Salié, Über die Kloostermanschen Summen $\mathcal{S}(u, v ; q)$, Math. Z. 34 (1932), no. 1, 91-109.

[19] I. E. Shpalinski, Exponential Sums in Coding Theory and Cryptography, Lecture Notes of Tutorial Lectures given at the Institute of Mathematics of the NUS, Singapore, July 23-26, 2001.

Department of Mathematics

SOGANG UNIVERSITY

SEOUL 121-742, KOREA

E-mail address: dskim@sogong.ac.kr 